\title{
Using Airflows Generated by Multicopter for Gas Detection in Midair
}

\author{
Kento Tanaka ${ }^{1}$, Saki Koguchi ${ }^{1}$, Ryohei Sato ${ }^{1}$, \\ Jane Pauline Ramos Ramirez ${ }^{2}$, Haruka Matsukura ${ }^{3}$, Hiroshi Ishida ${ }^{1}$ \\ ${ }^{1}$ Tokyo University of Agriculture and Technology, Tokyo, Japan, \\ ${ }^{2}$ University of the Philippines Diliman, Quezon City, Phillipines, \\ ${ }^{3}$ Osaka University, Osaka, Japana, \\ h_ishida@cc.tuat.ac.jp
}

\begin{abstract}
:
A new robotic gas sensing system that uses an airflow field generated by multicopters is proposed. Although a multi-rotor drone appears to be an attractive platform for gas sensing robots, strong airflow generated by its rotors when it flies poses a serious problem. Gas puffs leaked from the ground are never detected at the gas sensors on a multicopter because they all are blown away from the multicopter. In the proposed method, two multicopters connected by a string are used, and a gas sensor is placed at the midpoint of the string. The downward airflow generated by the rotors of the drone spreads radially after it reaches the ground. When two multicopters are connected, the airflows spreading radially from the two multicopters collide at the center, and are deflected in the upward direction. Gas puffs wafting near the ground surface between the two multicopters are carried by this upward airflow to the gas sensor. Thus, detection of the gas near the ground surface by the multicopters in midair is enabled. Experimental results are presented to show the soundness of the proposed method. A result of computational fluid dynamics simulation is also presented to show the possibility of adopting the same method in a single multicopter system.
\end{abstract}

Key words: multicopter, mobile robot, semiconductor gas sensor, gas source localization, landfill gas

\section{Introduction}

Mobile robots can be used as moving platforms for various gas sensing tasks. For example, a robot equipped with gas sensors can be used to monitor methane emission in landfill sites [1]. Methane is produced in a landfill site through bio-decomposition of solid waste. Periodical monitoring of methane gas concentration at a landfill site is often required by laws because methane is flammable and has strong greenhouse effect. A mobile robot can bring gas sensors to specified locations and autonomously collect sensor data to assess the total amount of methane emission in a landfill site.

Various gas sensing mobile robots have been reported over the past decades [2]. Although most of the robots reported so far are wheelbased, a multi-rotor drone appears to be a promising platform for gas sensing applications because of its high maneuverability over rough terrain [3]. However, a multicopter generates strong airflow in the downward direction when it flies. Any gas leaked from the ground is blown away from the multicopter, and no response is obtained from a gas sensor on board the multicopter.

Here we propose to utilize this problematic airflow for gas sensing. The airflow blown down from a multicopter spreads radially when it reaches the ground. If the two quadcopters are connected by a string as shown in Fig. 1, the airflow spreading from one of the quadcopters collide with the airflow from the other quadcopter and is deflected upward. If a gas

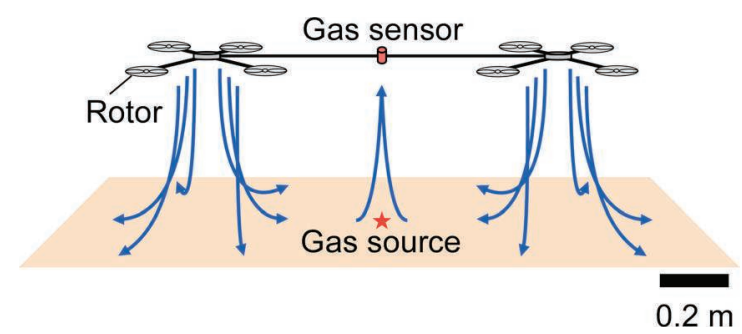

Fig. 1. Airflow field generated by two connected quadcopters. 


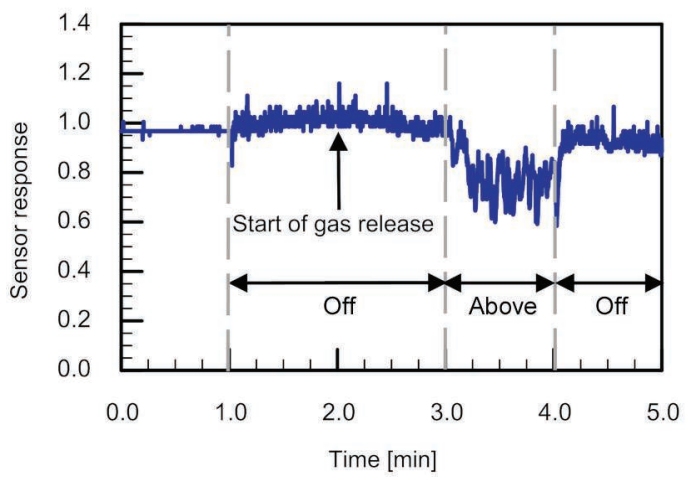

Fig. 2. Response curve of the gas sensor attached to the string that connects the two quadcopters.

sensor is attached at the midpoint of the string, this upward airflow brings gas puffs wafting near the ground surface to the gas sensor in midair. An experimental result is presented to show the feasibility of the proposed method. A result of computational fluid dynamics (CFD) simulation is also presented to show the possibility of adopting the same gas detection method in a single quadcopter system.

\section{Experiments}

Experiments were conducted in a closed indoor environment. Two quadcopters (AR.Drone 2.0, Parrot) were connected using a $1.7 \mathrm{~m}$ string, and a metal-oxide gas sensor (MiCS-5521, e2v technologies) was attached at the midpoint of the string. Fig. 2 shows the response curve of the gas sensor. The sensor response was defined as the ratio of the sensor resistance in gas to that in air. Its value decreases with the concentration of the detected gas. A tube releasing saturated ethanol vapor at a flow rate of $500 \mathrm{~mL} / \mathrm{min}$ was used as a gas source.

After one minute from the start of recording the sensor response, the rotors were activated to make the quadcopters hover at $0.5 \mathrm{~m}$ from the floor. Increased fluctuations in the sensor response shown in Fig. 2 were caused by exposing the sensor to the upward airflow from the floor, which was induced by activating the rotors. At two minutes from the start of the measurement, release for ethanol vapor was started. No change in the gas sensor response was observed at the beginning since the gas source was placed $1 \mathrm{~m}$ off to the side from the quadcopters. However, when the quadcopters were moved to hover directly above the gas source, the value of the sensor response decreased. The result shows that the ethanol vapor released from the gas source on the floor was successfully brought to the position of the gas sensor by the upward airflow as shown in Fig. 1.

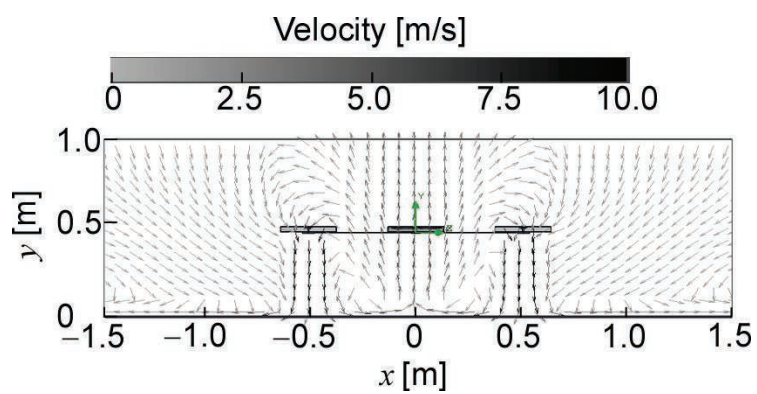

Fig. 3. Airflow field generated by a single quadcopter hovering near the ground.

\section{CFD Simulation}

Upward airflow similar to the one shown in Fig. 1 can be even generated by using a single quadcopter when it flies at a low altitude. Fig. 3 shows the result of CFD simulation for the airflow field generated by a quadcopter hovering at $0.5 \mathrm{~m}$ from the ground. The airflow field on the vertical cross section is shown in Fig. 3. The diagonal distance between the rotors was set to $1 \mathrm{~m}$. It can be seen in the figure that the airflow spreading from the two rotors are colliding at the midpoint and the upward airflow is generated. Detection of gas puffs drifting near the ground could be enabled by placing a gas sensor under the main body of the quadcopter.

\section{Conclusion}

A new robotic gas sensing system was proposed to detect gas puffs released from the ground by placing a gas sensor on a flying robot. Downward airflows generated by the rotors of two connected multicopters were used to whirl up the gas to the sensor in midair. The CFD simulation result shows that the same gas detection method can be used for a single quadcopter hovering at a low altitude. Future work will include testing prototype systems in the field. This work was supported in part by ACT-I, JST.

\section{References}

[1] V. Hernandez Bennetts, A. J. Lilienthal, P. P. Neumann, M. Trincavelli, Mobile Robots for Localizing Gas Emission Sources on Landfill Sites: Is Bio-inspiration the Way to Go?, Front. Neuroroeng. 4, article 20 (2012); doi: 10.3389/fneng.2011.00020

[2] H. Ishida, Y. Wada, H. Matsukura, Chemical Sensing in Robotic Applications: A Review, IEEE Sensors J. 12, 3163-3173 (2012); doi: 10.1109/JSEN.2012.2208740

[3] P. P. Neumann, V. Hernandez Bennetts, A. J. Lilienthal, M. Bartholmai, J. H. Schiller, Gas Source Localization with a Micro-drone using bioinspired and particle filter-based algorithms, Adv. Robot. 27, 725-738 (2013); doi: $10.1080 / 01691864.2013 .779052$ 